\title{
Specific tail swelling pattern in hypo-osmotic solution as a predictor of DNA fragmentation status in human spermatozoa
}

\author{
Sung Woo Kim ${ }^{1 *}$, Eun Jee Nho ${ }^{2 *}$, Joong Yeup Lee ${ }^{3}$, Byung Chul Jee ${ }^{1,2}$ \\ 'Department of Obstetrics and Gynecology, Seoul National University College of Medicine, Seoul; ${ }^{2}$ Department of Obstetrics and Gynecology, Seoul \\ National University Bundang Hospital, Seongnam; ${ }^{3}$ Hamchoon Women's Clinic, Seoul, Korea
}

Objective: The aim of this study was to investigate DNA fragmentation status in human spermatozoa according to specific tail swelling patterns determined via hypo-osmotic swelling test (HOST).

Methods: Frozen semen samples from 21 healthy donors were thawed and prepared by the swim-up technique for use in intracytoplasmic sperm injection. The semen samples were treated for 5 minutes as part of the HOST procedure and then underwent the sperm chromatin dispersion test using a Halosperm kit. DNA fragmentation status (large halo, medium halo, small halo, no halo, or degraded) and the specific tail swelling pattern ("a"-" $\mathrm{g}$ ") were assessed at the level of a single spermatozoon. A total of 42,000 spermatozoa were analyzed, and the percentage of spermatozoa without DNA fragmentation (as evidenced by a large or medium halo) was assessed according to the specific tail swelling patterns observed.

Results: The HOST examinations showed that $>93 \%$ of spermatozoa across all types displayed no DNA fragmentation. The percentage of spermatozoa without DNA fragmentation was 100\% in type "d", $98.67 \%$ in type " $g$ ", and $98.17 \%$ in type " $f$ " spermatozoa.

Conclusion: We found that the type " $d$ " spermatozoa displayed no DNA fragmentation, but the other types of spermatozoa also displayed very low rates of DNA fragmentation. This result may be associated with the processing of the spermatozoa by density gradient centrifugation and the swim-up technique.

Keywords: DNA fragmentation; Infertility; Sperm head; Spermatozoa

\section{Introduction}

When performing intracytoplasmic sperm injection (ICSI), spermatozoa are typically selected based on their motility and morphology as determined by a visual inspection under a light microscope. How-

Received: July 23, 2019 · Revised: August 28, 2019 A Accepted: September 5, 2019 Corresponding author: Byung Chul Jee

Department of Obstetrics and Gynecology, Seoul National University Bundang Hospital, Seoul National University College of Medicine, 82 Gumi-ro 173beongil, Bundang-gu, Seongnam 13620, Korea

Tel:+82-31-787-7254 Fax:+82-31-787-4054 E-mail: blasto@snubh.org

*These authors contributed equally to this study.

**This work was supported by grant no. 06-2018-097 from the Seoul National University Bundang Hospital Research Fund.

This is an Open Access article distributed under the terms of the Creative Commons Attribution Non-Commercial License (http://creativecommons.org/licenses/by-nc/4.0/) which permits unrestricted non-commercial use, distribution, and reproduction in any medium, provided the original work is properly cited. ever, this conventional procedure still allows the selection of spermatozoa with DNA fragmentation. A high level of sperm DNA fragmentation (SDF) has been reported to be associated with a decreased fertilization rate, poor embryonic development, and poor pregnancy outcomes [1-7]. Selection of spermatozoa that display either no DNA fragmentation or the least amount of fragmentation possible may be an important component of ICSI in the future and may improve embryo quality and reproductive outcomes.

Several methods can be used to increase the prevalence of spermatozoa with low SDF levels in a given sample. These include sperm preparation methods, such as the swim-up method and density gradient centrifugation, as well as several sperm sorting methods, including annexin V binding-based magnetic-activated cell sorting and hyaluronic acid binding-based physiological ICSI [8]. However, methods of selecting a single live spermatozoon without DNA dam- 
age are currently unavailable.

Several tests to examine SDF levels exist, such as the terminal deoxynucleotidyl transferase dUTP nick end labeling (TUNEL) assay, the comet assay, and sperm chromatin dispersion (SCD). However, spermatozoa tested using these techniques are not viable because they are processed by fixation and staining during the examination and thus are not useful for clinical purposes. Therefore, noninvasive techniques to select a single live spermatozoon without DNA damage are needed.

The hypo-osmotic swelling test (HOST) is a simple vitality test used to assess the tail membrane function of spermatozoa $[9,10]$. When semen samples are incubated in a hypo-osmotic solution (150 $\mathrm{mOsm})$, the tails of the sperm in solution may or may not become swollen. Among sperm tails that do swell, the specific swelling pattern can be used to classify the spermatozoon as one of six types. The presence of any swelling (as denoted by spermatozoa types " $b$ "- " $\mathrm{g}$ ") represents healthy cell membrane function, and these spermatozoa are thus considered to be viable. The lower reference limit for vitality (i.e., membrane-intact spermatozoa) is 58\% [10]. The tested spermatozoa can be used directly in ICSI because the test does not harm the head or neck of the spermatozoa.

The HOST score, or sperm vitality as measured by the HOST in raw semen, has been reported to be correlated with sperm motility, percentage of normal form, and SDF level (as measured by acridine orange staining) [11]. In that study, when individual spermatozoa were sequentially treated by HOST and then TUNEL staining, types "d", "e", and " $f$ " spermatozoa showed significantly lower SDF levels than types "a" and " $g$ " spermatozoa. In another study, the SDF level (as assessed by the TUNEL method) was lowest in type "b" spermatozoa (mean, 3.9\%), which was significantly lower than in type "a" (mean, 16.8\%) or "g" spermatozoa (mean, 13\%) [12]. In that report, type "d" spermatozoa also displayed a significantly lower SDF level (mean, 5\%) than type "a". However, in those studies, spermatozoa were tested in raw semen that had not been prepared for ICSI use. Visualization of spermatozoa after TUNEL staining requires expensive immunofluorescence equipment. In contrast, SCD testing using a Halosperm kit (Halotech DNA, Madrid, Spain) does not require immunofluorescence equipment.

In the present study, frozen semen samples were thawed and prepared by swim-up for ICSI use and were sequentially treated using the HOST and the SCD tests. The DNA fragmentation status was assessed at the level of a single spermatozoon, and the SDF level was assessed according to the specific tail swelling pattern observed.

\section{Methods}

Semen samples were obtained from 21 healthy donors between
2008 and 2014. The semen samples were processed by density gradient centrifugation (SAGE gradient kit, 40\%/80\%; CooperSurgical, Copenhagen, Denmark) and were rapidly frozen using the liquid nitrogen $\left(\mathrm{LN}_{2}\right)$ vapor method. A commercial yolk buffer was used as a basic freezing medium (Difco Egg Yolk Enrichment 50\%; BD, Franklin Lakes, NJ, USA). The median age of the male donors at the time of semen collection was 27 years (interquartile range, 26-29 years). Unfortunately, no information was known regarding the marriage or fertility status of the sperm donors, their medical or surgical history, or their prescribed medications, if any. At the time of semen collection, informed consent was obtained from all subjects for the later therapeutic or experimental use of the samples.

A prospective study was performed from 2016 to 2017. The Institutional Review Board of Hamchoon Women's Clinic and Seoul National University Bundang Hospital approved this study (IRB No. B-1804463-301). The semen samples were thawed by rapidly immersing the straws in a water bath at $37^{\circ} \mathrm{C}$ for 5 minutes. The thawed samples were mixed with $3 \mathrm{~mL}$ of Ham's F-10 solution containing 10\% human serum albumin (HSA; Irvine Scientific, Santa Ana, CA, USA). The basic sperm concentration and motility were then assessed using motion analysis equipment (SAIS-PLUS 10.1; Medical Supply Co., Seoul, Korea). Strict criteria for the definition of normal sperm were used during the morphological assessment. According to the 2010 guidelines from the World Health Organization (WHO) [10], a sperm count $\geq 15$ million/mL, motility $\geq 40 \%$, and a percentage of normal form $\geq 4 \%$ were defined as normal. After thawing, the semen displayed a median sperm count of 20 million/mL (interquartile range, 10-33 million/ $\mathrm{mL}$ ), a median total motility of $30.8 \%$ (interquartile range, $19.2 \%$ $51.8 \%$ ) and a median percentage of normal form of $6.7 \%$ (interquartile range, $3.9 \%-8.5 \%)$.

The semen samples were centrifuged three times at $250 \times g$ for 5 minutes after mixing with $3 \mathrm{~mL}$ of Ham's F-10 solution containing $10 \%$ HSA. The semen samples were then processed using the swimup method in the same procedure used for the preparation for ICSI in our center; $0.5-1 \mathrm{~mL}$ of Ham's F-10 containing $10 \% \mathrm{HSA}$ was gently layered on the pellet and then incubated at $37^{\circ} \mathrm{C}$ in a $5 \% \mathrm{CO}_{2}$ atmosphere for 30 minutes. The supernatant $(0.5 \mathrm{~mL})$ was transferred to a conical tube and was then used for HOST.

HOST was performed using the method described previously $[10,11]$. A hypo-osmotic solution was prepared by dissolving $0.735 \mathrm{~g}$ of sodium citrate dihydrate and $1.351 \mathrm{~g}$ of D-fructose in $100 \mathrm{~mL}$ of purified water. The final solution was set at $150 \mathrm{mOsm}$ and was then frozen at $-20^{\circ} \mathrm{C}$ until later use. For each semen sample, $0.1 \mathrm{~mL}$ of semen was added to $1 \mathrm{~mL}$ of warmed hypo-osmotic solution and incubated at $37^{\circ} \mathrm{C}$ for 5 minutes. In HOST, 30 minutes of incubation is suggested for routine diagnostic procedures, but 5 minutes of incubation is recommended when spermatozoa are to be processed for 
therapeutic use [10].

For the SCD test, a Halosperm kit was used as described previously $[13,14]$. After assessment of tail swelling, the semen samples $(25 \mu \mathrm{L})$ were mixed with pre-warmed agarose gel and were then dropped onto slides. The slides were covered with a glass coverslip and were kept for 5 minutes at $4^{\circ} \mathrm{C}$ in a refrigerator to create a microgel with implanted sperm. The coverslip was then removed, and the slides were immersed into a prepared acid solution $(80 \mu \mathrm{L}$ of $\mathrm{HCl}$ in $10 \mathrm{~mL}$ of distilled water) for 7 minutes at room temperature (RT). The slides were then transferred to the tray with a lysis solution and incubated for 25 minutes at RT. The slides were rinsed with distilled water for 5 minutes, followed by dehydration in increasing concentrations of ethanol (70\%, 90\%, and 100\%, for 2 minutes each). After drying, the slides were stained with Diff-Quik (Baxter Diagnostics Inc., McGaw Park, IL, USA), rinsed under tap water, and were allowed to dry at RT.

Each slide was examined under the light microscope at $\times 400$ or $\times 1,000$ magnification, and the tail swelling patterns and head halo patterns were simultaneously assessed for 200 spermatozoa per slide (Figure 1). Ten slides were prepared for each participant; thus, the final count of spermatozoa was 2,000 per person. Each sperm head was counted as either a large halo, medium halo, small halo, no halo, or degraded; a spermatozoon was classified as having a large halo if it produced a halo with a thickness equal to or greater than the length of the minor diameter of the core, as having a medium halo if the spermatozoon produced a halo with a thickness smaller than the length of the minor diameter of the core and greater than one-third of the minor diameter of the core, and as having a small halo if the spermatozoon produced a halo with a thickness equal to or smaller than one-third of the minor diameter of the core. Spermatozoa with a small halo or no halo, and those classified as degraded, were considered spermatozoa with fragmented DNA.

All statistical analyses were performed using IBM SPSS ver. 22.0 (IBM Corp., Armonk, NY, USA). The Spearman correlation test was used to evaluate the correlations between parameters. The results were considered significant when the $p$-value was $<0.05$.

\section{Results}

In this study, the median HOST score was $16.7 \%$ (interquartile range, $10.4 \%-20.7 \%$ ), and the median SDF level was $1.40 \%$ (interquartile range, $0.65 \%-2.83 \%)$. The HOST score was not correlated with the age of the man, sperm concentration, total motility, or the percentage of normal form. The SDF level was not correlated with the age of the man, sperm concentration, or motility, but it was negatively correlated with the percentage of normal form (Spearman coefficient of rank correlation, $r=-0.473 ; p=0.034)$. The HOST score
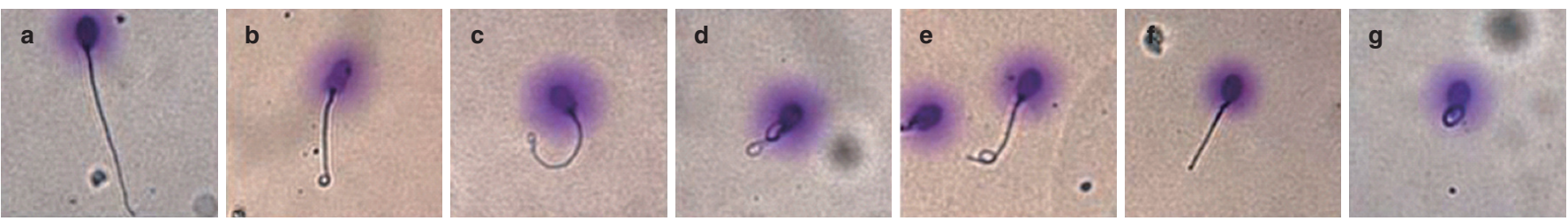

Figure 1. Representative photographs showing sperm heads with large halos after the sperm chromatin dispersion test. Each letter (a-g) corresponds to the specific tail swelling type after the hypo-osmotic swelling test.

Table 1. The number of spermatozoa exhibiting each of the five head halo patterns and categorized as each of the seven types after the HOST

\begin{tabular}{|c|c|c|c|c|c|c|c|c|}
\hline \multirow[b]{2}{*}{ HOST type } & \multicolumn{7}{|c|}{ Head halo pattern } & \multirow[b]{2}{*}{$\begin{array}{c}\text { Spermatozoa } \\
\text { without DF (\%) }\end{array}$} \\
\hline & Large halo & Medium halo & Small halo & No halo & Degraded & Total & $\begin{array}{c}\text { Large } \\
\text { halo+medium } \\
\text { halo }\end{array}$ & \\
\hline a & 30,316 & 3,380 & 505 & 154 & 49 & 34,404 & 33,696 & 97.94 \\
\hline $\mathrm{b}$ & 884 & 242 & 25 & 2 & 0 & 1,153 & 1,126 & 97.66 \\
\hline c & 288 & 119 & 11 & 0 & 0 & 418 & 407 & 97.37 \\
\hline$d$ & 60 & 24 & 0 & 0 & 0 & 84 & 84 & 100.00 \\
\hline e & 130 & 78 & 7 & 1 & 0 & 216 & 208 & 96.3 \\
\hline$f$ & 1,439 & 489 & 30 & 5 & 1 & 1,964 & 1,928 & 98.17 \\
\hline $\mathrm{g}$ & 2,685 & 1,026 & 49 & 1 & 0 & 3,761 & 3,711 & 98.67 \\
\hline Total & 35,802 & 5,358 & 627 & 163 & 50 & 42,000 & 41,160 & 98.00 \\
\hline
\end{tabular}

The data above were pooled from 21 men.

HOST, hypo-osmotic swelling test; DF, DNA fragmentation.

a)The pooled percentages of spermatozoa without DF by type after the HOST are listed in the last column. 
was not correlated with the SDF level.

The results of a pooled analysis after sequential treatment using the HOST and the SCD test are presented in Table 1. Across all types of spermatozoa as classified by the HOST procedure, $>93 \%$ of spermatozoa lacked DNA fragmentation (i.e., had a large or medium halo), and no statistical significance was noted among the seven types from HOST. However, the proportion of spermatozoa without DNA fragmentation was highest in type " $d$ " (100\%), followed by type “g” (98.67\%) and type “f” (98.17\%).

\section{Discussion}

In the present study, type " $d$ " spermatozoa displayed no DNA fragmentation (i.e., all type " $d$ " spermatozoa exhibited a large or medium halo). Unfortunately, type " $d$ " spermatozoa were rare $(0.2 \%$ of the total pool of spermatozoa), which is consistent with the findings of Bassiri et al. [12]. The percentage of spermatozoa without DNA fragmentation was $98.67 \%$ in type " $\mathrm{g}$ " spermatozoa and $98.17 \%$ in type "f" spermatozoa. Since type " $g$ " and type "f" spermatozoa were not rare (8.9\% and $4.7 \%$ of the total pool of spermatozoa, respectively), it would be reasonable to choose type " $g$ " or type "f" spermatozoa for ICSI use, assuming no type " $d$ " spermatozoa are available.

In one study, types " $d$ ", "e", and "f" spermatozoa showed a significantly lower SDF level (as assessed by the TUNEL method) than types "a" or " $g$ " spermatozoa [11]. In another study, the SDF level (as assessed by the TUNEL method) was lowest in type "b" (mean 3.9\%), which was significantly lower than that found in type "a" (mean, $16.8 \%)$ or type " $g$ "(mean, 13\%) spermatozoa [12]. In that report, type " $d$ " spermatozoa also displayed a significantly lower SDF level (mean $5 \%)$ than type "a".

In those reports, type " $g$ " spermatozoa displayed a relatively high SDF level; however, we found that type " $g$ " spermatozoa displayed a relatively low SDF level $[11,12]$. The explanation for this difference in findings is not known for certain, but possible reasons could be that we used a different sperm processing and different assay method for SDF. Nonetheless, type " $d$ " spermatozoa showed a good SDF level in our study and in both other reports.

The lower reference limit for the HOST score is 58\% [10]. In the present study, however, the median value of the HOST score was $16.7 \%$ (interquartile range, 10.4\%-20.7\%). This lower median HOST score observed in our study might have been related to our relatively short incubation time of 5 minutes. The WHO manual recommends 30 minutes of incubation for routine diagnostic procedures, but it recommends only 5 minutes of incubation for therapeutic purposes [10]. A shorter incubation time for the HOST procedure would result in less swelling of the spermatozoa tail; thus, the HOST score is expected to be lower after 5 minutes of incubation than after 30 min- utes of incubation.

Additionally, the median SDF level was very low in the present study (1.40\%; interquartile range, $0.65 \%-2.83 \%)$. We believe that the semen samples used were relatively good, as they were obtained from young, healthy donors. Furthermore, the semen samples were processed by density gradient centrifugation before freezing and were processed by the swim-up method after thawing.

In our experiment, we could not simultaneously evaluate the sperm head morphology and the tail swelling pattern of an individual spermatozoon because the sperm head was already swollen due to the SCD test. However, in a previous study, types " $d$ " and " $c$ " spermatozoa showed a significantly higher percentage of normal form than type "a" spermatozoa [15]. In another study conducted by the same researchers, the percentage of normal form was significantly higher in type " $d$ " spermatozoa than in the other types [12]. In that report, type " $d$ " spermatozoa, followed by type " $c$ ", exhibited the highest quality in terms of early and late apoptosis, nuclear maturity, and membrane integrity [12].

A limitation of the present study is that the spermatozoa were obtained only from donors for the purpose of artificial insemination. Additionally, all of the samples were frozen. Further studies will be required to ascertain the relationship between the HOST pattern and SDF level in an infertile population and/or in fresh, unfrozen spermatozoa.

A sibling oocyte study in couples with severe male infertility revealed that embryos exhibited better quality and a higher implantation rate when type "c", " $d$ ", or "e" spermatozoa were preferentially selected, compared to the results of conventional selection [16]. We recommend routine HOST during ICSI and subsequent selection of the specific types of spermatozoa that have been found to have the lowest SDF level. However, our recommendation should be further confirmed by a clinical trial. HOST is a simple and convenient technique that does not destroy the structure of the spermatozoon. The application of HOST may be a valuable tool in the routine identification and selection of viable, DNA-intact individual spermatozoa for ICSI.

\section{Conflict of interest}

No potential conflict of interest relevant to this article was reported.

\section{ORCID}

Sung Woo Kim https://orcid.org/0000-0003-4689-1323

Eun Jee Nho

https://orcid.org/0000-0002-8214-1434

Joong Yeup Lee https://orcid.org/0000-0002-1536-3811

Byung Chul Jee https://orcid.org/0000-0003-2289-6090 


\section{Author contributions}

Conceptualization: SWK, JYL, BCJ. Data curation: SWK, EJN, BCJ. Formal analysis: SWK, BCJ. Funding acquisition: JYL, BCJ. Methodology: all authors. Project administration: JYL, BCJ. Visualization: SWK, EJN, BCJ. Writing - original draft: all authors. Writing - review \& editing: SWK, EJN, BCJ.

\section{References}

1. Carrell DT, Liu L, Peterson CM, Jones KP, Hatasaka HH, Erickson L, et al. Sperm DNA fragmentation is increased in couples with unexplained recurrent pregnancy loss. Arch Androl 2003;49:49-55.

2. Saleh RA, Agarwal A, Sharma RK, Said TM, Sikka SC, Thomas AJ Jr. Evaluation of nuclear DNA damage in spermatozoa from infertile men with varicocele. Fertil Steril 2003;80:1431-6.

3. Henkel R, Hajimohammad M, Stalf T, Hoogendijk C, Mehnert C, Menkveld $\mathrm{R}$, et al. Influence of deoxyribonucleic acid damage on fertilization and pregnancy. Fertil Steril 2004;81:965-72.

4. Borini A, Tarozzi N, Bizzaro D, Bonu MA, Fava L, Flamigni C, et al. Sperm DNA fragmentation: paternal effect on early post-implantation embryo development in ART. Hum Reprod 2006;21: 2876-81.

5. Evenson D, Wixon R. Meta-analysis of sperm DNA fragmentation using the sperm chromatin structure assay. Reprod Biomed Online 2006;12:466-72.

6. Li Z, Wang L, Cai J, Huang H. Correlation of sperm DNA damage with IVF and ICSI outcomes: a systematic review and meta-analysis. J Assist Reprod Genet 2006;23:367-76.

7. Robinson L, Gallos ID, Conner SJ, Rajkhowa M, Miller D, Lewis S, et al. The effect of sperm DNA fragmentation on miscarriage rates: a systematic review and meta-analysis. Hum Reprod 2012;27:2908-17.
8. Nadalini M, Tarozzi N, Di Santo M, Borini A. Annexin V magneticactivated cell sorting versus swim-up for the selection of human sperm in ART: is the new approach better then the traditional one? J Assist Reprod Genet 2014;31:1045-51.

9. Jeyendran RS, Van der Ven HH, Perez-Pelaez M, Crabo BG, Zaneveld LJ. Development of an assay to assess the functional integrity of the human sperm membrane and its relationship to other semen characteristics. J Reprod Fertil 1984;70:219-28.

10. World Health Organization. WHO laboratory manual for the examination and processing of human semen. 5th ed. Geneva: World Health Organization; 2010.

11. Stanger JD, Vo L, Yovich JL, Almahbobi G. Hypo-osmotic swelling test identifies individual spermatozoa with minimal DNA fragmentation. Reprod Biomed Online 2010;21:474-84.

12. Bassiri F, Tavalaee M, Shiravi AH, Mansouri S, Nasr-Esfahani MH. Is there an association between HOST grades and sperm quality? Hum Reprod 2012;27:2277-84.

13. Tandara M, Bajic A, Tandara L, Bilic-Zulle L, Sunj M, Kozina V, et al. Sperm DNA integrity testing: big halo is a good predictor of embryo quality and pregnancy after conventional IVF. Andrology 2014;2:678-86.

14. Anifandis G, Bounartzi T, Messini Cl, Dafopoulos K, Markandona $\mathrm{R}$, Sotiriou $\mathrm{S}$, et al. Sperm DNA fragmentation measured by Halosperm does not impact on embryo quality and ongoing pregnancy rates in IVF/ICSI treatments. Andrologia 2015;47:295-302.

15. Bassiri F, Tavalaee M, Nasr Esfahani MH. Correlation between different patterns of hypo-osmotic swelling and sperm functional tests. Int J Fertil Steril 2013;7:193-8.

16. Charehjooy N, Najafi MH, Tavalaee M, Deemeh MR, Azadi L, Shiravi $\mathrm{AH}$, et al. Selection of sperm based on hypo-osmotic swelling may improve ICSI outcome: a preliminary prospective clinical trial. Int J Fertil Steril 2014;8:21-8. 